\title{
Ripple Frequency Determined via a Novel Algorithm is Associated with Atrial Fibrillation Termination and Freedom from Atrial Fibrillation
}

Daniel Melby ${ }^{1}$, Refael Itah $^{2}$, David Benditt ${ }^{3}$, Venkatakrishna Tholakanahalli ${ }^{4}$, Raed Abdelhadi $^{1}$, Jay Sengupta ${ }^{5}$, Charles Gornick ${ }^{6}$, JoEllyn Moore ${ }^{7}$, Manjunath Pai ${ }^{1}$, and John Zakaib $^{7}$

${ }^{1}$ Abbott Northwestern Hospital

${ }^{2}$ Biosense Webster Inc

${ }^{3}$ University of Minnesota Medical Center

${ }^{4}$ Division of Cardiology, VA Medical Center, Minneapolis, MN \& University of Minnesota

${ }^{5}$ Minneapolis Heart Institute at Abbott Northwestern Hospital

${ }^{6}$ Minneapolis Heart Institute Foundation at Abbott Northwestern Hospital

${ }^{7}$ Minneapolis Heart Institute Foundation

December 5, 2021

\begin{abstract}
Background: Persistent atrial fibrillation (PsAF) is a complex arrhythmia and achieving a high rate of freedom from recurrence

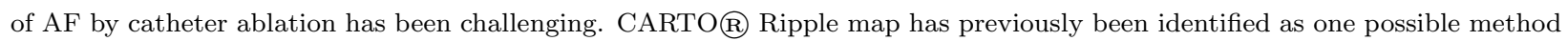
to improve ablation outcomes. Objective: To evaluate the relationship of AF termination and 18-month freedom from AF with Ripple Frequency measured via a novel CARTO@ software algorithm. Methods: PsAF patients who underwent first time ablation were included. PV antral isolation was performed followed by locations with visually identified fast Ripple activations until AF termination. Patients were followed for 18 months. Retrospective analysis was performed using a novel CARTO $\mathbb{R}$ software algorithm to analyze Ripple Frequency. The Ripple Frequency algorithm quantifies amplitude changes in the bipolar electrogram over time. Results: 82 maps from 54 patients (mean age 65.4, 67\% male) were analyzed. The top quartile of Ripple Frequency corresponded to a visual reference with $96.1 \%$ sensitivity and $84.7 \%$ specificity. AF terminated during ablation in 90.7\% of patients: PV antrum alone (14.8\%), or PV plus non-antral sites (85.2\%). The top quartile of Ripple frequency was present in non-antral sites associated with AF termination with an $89.6 \%$ sensitivity and $87.7 \%$ specificity. After 18 months and a mean of 1.2 ablations, $53 / 54$ patients (98.1\%) were free of $\mathrm{AF}$ and $85.2 \%$ were free of any atrial arrhythmia. Conclusion: Automated analysis of CARTO@, Ripple Frequency demonstrated good sensitivity and specificity for detecting atrial regions in PsAF where ablation is associated with AF termination and freedom from AF after 18 months.
\end{abstract}

\section{Hosted file}

Ripple Frequency Manuscript 11-22-21.docx available at https://authorea.com/users/449642/ articles/548132-ripple-frequency-determined-via-a-novel-algorithm-is-associated-withatrial-fibrillation-termination-and-freedom-from-atrial-fibrillation 